\title{
Article
}

\section{Effects of partial internal biliary diversion on long-term outcomes in patients with progressive familial intrahepatic cholestasis: experience in 44 patients}

Foroutan, Hamid Reza, Bahador, Ali, Ghanim, Sultan Mohsin, Dehghani, Seyed Mohsen, Anbardar, Mohammad Hossein, Fattahi, Mohammad Reza, Forooghi, Mehdi, Azh, Omidreza, Tadayon, Ali and Sherafat, Alireza

Available at http://clok.uclan.ac.uk/32828/

Foroutan, Hamid Reza, Bahador, Ali, Ghanim, Sultan Mohsin, Dehghani, Seyed Mohsen, Anbardar, Mohammad Hossein, Fattahi, Mohammad Reza, Forooghi, Mehdi, Azh, Omidreza, Tadayon, Ali et al (2020) Effects of partial internal biliary diversion on long-term outcomes in patients with progressive familial intrahepatic cholestasis: experience in 44 patients. Pediatric Surgery International . ISSN 0179-0358

It is advisable to refer to the publisher's version if you intend to cite from the work. http://dx.doi.org/10.1007/s00383-020-04641-z

For more information about UCLan's research in this area go to

http://www.uclan.ac.uk/researchgroups/ and search for <name of research Group>.

For information about Research generally at UCLan please go to http://www.uclan.ac.uk/research/

All outputs in CLoK are protected by Intellectual Property Rights law, including Copyright law. Copyright, IPR and Moral Rights for the works on this site are retained by the individual authors and/or other copyright owners. Terms and conditions for use of this material are defined in the policies page. 


\section{Effects of partial internal biliary diversion on long-term outcomes in patients with progressive familial intrahepatic cholestasis : Experience in $\mathbf{4 4}$ patients}

Hamid Reza Foroutan ${ }^{1}$, Ali Bahador ${ }^{2}$, Sultan Mohsin Ghanim², Seyed Mohsen Dehghani ${ }^{4}$, Mohammad Hossein Anbardar ${ }^{5}$, mohammad reza fattahi ${ }^{6}$, mehdi forooghi ${ }^{2}$, Omidreza Azh ${ }^{2}$, Ali Tadayon ${ }^{2}$, Alireza Sherafat ${ }^{7}$,Amir Arsalan Yaghoobi ${ }^{2,3}$, Mohammad Ali Ashraf ${ }^{2,3 *}$

Affiliations:

1. Laparoscopy Research Center, Shiraz University of Medical Sciences, Shiraz, Iran

2. Department of Pediatric Surgery, Nemazee Hospital, Shiraz University of Medical Sciences, Shiraz, Iran

3. Student research committee, Shiraz University of Medical Sciences, Shiraz, Iran

4. Gastroenterohepatology Research Center, Department of Pediatric Gastroenterology, Nemazee Hospital, Shiraz University of Medical Sciences, Shiraz, Iran

5. Department of Pathology, Shiraz Medical School, Shiraz University of Medical Sciences, Shiraz, Iran

6. Department of Gastroenterology, Nemazee Hospital , Shiraz University of Medical Sciences, Shiraz, Iran

7. School of Medicine, University of Central Lancashire, England

First author:

Hamid Reza Foroutan

Pediatric surgery ward, Nemazee hospital, Shiraz University of Medical Sciences, Zand St., 7132326645 , Shiraz, Iran

Tel/Fax: +98 7136474332

Email address: Forotanh@yahoo.com

Corresponding author* :

Mohammad Ali Ashraf

Student Research Committee, Shiraz University of Medical Sciences, Shiraz, Iran

Zand St., 71 32326645, Shiraz, Iran

Tel/Fax: +98 7132326645

Email address: $\underline{\text { mohammadali1374.ashraf@gmail.com }}$ 


\section{Abstract}

Purpose: Progressive familial intrahepatic cholestasis (PFIC) is a hereditary disease characterized by cholestasis, which may cause jaundice, severe pruritus, and cirrhosis in the later stages. By the invention of biliary diversion methods, these patients were prevented from undergoing liver transplant. Using biliary diversion techniques, the entero-hepatic cycle was interrupted. This lowers the bile acid pool and resolves the pruritus.

Herein, we report 44 cases of PFIC who underwent partial internal biliary diversion (PIBD) and long-term followup of these children. This comprises the largest case series of PIBD.

Methods: All patients were diagnosed by liver biopsy as PFIC before the operation. All underwent cholecysto colic bypass by jejunal interposition due to severe pruritus unresponsive to medication. Laboratory blood tests, sonography, and physical exam were done before and after the operation once every three months. Besides, a questionnaire was designed to ask the patients about the symptoms after the operation, and a pruritus score was measured using the 5D-itch scale.

Results: 44 children ( 25 boys, 19 girls), between 1.75 to 27.5 years (at the time of this study) were followed for a median period of 54 months. Age at operation ranged from 2 months to 18 years, with a median of 29 months. Of these children, 14 were lost to follow up. Results showed a significant decrease in pruritus and sleep disturbance after the surgery $(p<0.001)$. Also, jaundice decreased from $82.1 \%$ before to $7.1 \%$ following the surgery. $50 \%$ of the patients became medication-free at follow-up.

Conclusion: PIBD is a safe procedure which helps non-cirrhotic children preserve their liver function. Therefore PIBD prevents them from undergoing liver transplant. Effective results were achieved in terms of severe pruritus and jaundice, and children were able to regain their sleep patterns. It also avoided external stoma, which is more convenient from the patient's point of view. 